\title{
Efficient isolation of specific genomic regions by insertional chromatin immunoprecipitation (iChIP) with a second-generation tagged LexA DNA-binding domain
}

\author{
Toshitsugu Fujita, Hodaka Fujii* \\ Combined Program on Microbiology and Immunology, Research Institute for Microbial Diseases, Osaka University, Osaka, Japan \\ Email: "hodaka@,biken.osaka-u.ac.jp
}

Received 18 June 2012; revised 20 July 2012; accepted 2 August 2012

\begin{abstract}
Comprehensive understanding of mechanisms of epigenetic regulation requires identification of molecules bound to genomic regions of interest in vivo. We have developed a novel method, insertional chromatin immunoprecipitatin (iChIP), to isolate specific genomic regions retaining in vivo molecular interaction in order to perform non-biased identification of interacting molecules. Here, we developed a second-generation tagged LexA DNA-binding domain, $3 \times$ FNLDD, for the iChIP analysis. $3 \times$ FNLDD consists of $3 \times$ FLAG tags, a nuclear localization signal (NLS), the DNA-binding domain (DB) and the dimerization domain of the LexA protein. Expression of $3 \times$ FNLDD can be detected by immunoblot analysis as well as flowcytometry. We showed that iChIP using $3 \times$ FNLDD is able to consistently isolate more than $10 \%$ of input genomic DNA, several-fold more efficient compared to the first-generation tagged LexA DB. $3 \times$ FNLDD would be a useful tool to perform the iChIP analysis for locus-specific biochemical epigenetics.
\end{abstract}

Keywords: Insertional Chromatin Immunoprecipitation; iChIP; LexA; FLAG Tag

\section{INTRODUCTION}

Epigenetic regulation of eukaryotic genomic regions is mediated by molecular complexes in the context of chromatin [1]. We recently developed insertional chromatin immunoprecipitation (iChIP), which is a method to biochemically isolate a genomic region of interest which retains molecular interaction [2]. The scheme of iChIP is as follows: 1) A repeat of the recognition sequence of an exogenous DNA-binding protein such as LexA is inserted into the genomic region of interest in the cell to be analyzed. 2) The DNA-binding domain (DB) of the ex-

${ }^{*}$ Corresponding author. ogenous DNA-binding protein is fused with a $\operatorname{tag}(\mathrm{s})$ and a nuclear localization signal (NLS)(s) and expressed into the cell to be analyzed. 3) The resultant cell is stimulated, if necessary, and crosslinked with formaldehyde or other crosslinkers. 4) The cell is lysed, and the crosslinked DNA is fragmented by sonication. 5) The complexes including the exogenous DB are immunoprecipitated with an antibody against the tag. 6) The isolated complexes retain molecules interacting with the genomic region of interest. Reverse crosslinking and subsequent purification of DNA, RNA, proteins, or other molecules allows their identification and characterization. By using iChIP, we succeeded in directly identifying protein and RNA components of an insulator, which functions as boundaries of chromatin domains [3], showing that iChIP is a powerful tool for elucidation of molecular mechanisms of epigenetic regulation. In order to easily identify molecular interaction in vivo, however, more efficient isolation of specific genomic regions of interest is necessary.

To this end, here, we developed a second-generation tagged LexA DNA-binding domain, $3 \times$ FNLDD, to perform the iChIP analysis more efficiently. $3 \times$ FNLDD consists of $3 \times$ FLAG tags, an NLS, DB and the dimerization domain of the LexA protein. Expression of $3 \times$ FNLDD can be detected by immunoblot analysis as well as flowcytometry. We showed that iChIP using $3 \times$ FNLDD is able to consistently isolate more than $10 \%$ of input of specific genomic regions, several-fold more efficient compared to the first-generation tagged LexA DB. $3 \times$ FNLDD would be useful for the iChIP analysis of specific genomic regions to perform their biochemical analysis.

\section{MATERIALS AND METHODS}

\subsection{Plasmid Construction}

To construct $3 \times \mathrm{FNLDD} / \mathrm{pCMV}-7.1$, the DNA sequence encoding NLS-LexA DB was cleaved from FCNLD/ pMIR [2] with BamH I and Not I, blunted, and inserted 
into p3XFLAG-CMV-7.1 (Sigma-Aldrich) that was cleaved with $\mathrm{Bgl}$ II and blunted to generate $3 \times \mathrm{FNLD} /$ pCMV-7.1. Subsequently, the DNA sequence encoding DB and the dimerization domain of LexA was amplified with the LexA-N (26081) (5'-cccttCCTGAGGGAATG AAAGCGTTAACG-3') and LexA-C w/D (26628) (5'-aa atgtcgaCTACAGCCAGTCGCCGTTGCG-3') primers using pBTM116 [4] as template. The PCR product was cleaved with Mlu I and Sal I and inserted into Mlu I- and Sal I-cleaved $3 \times$ FNLD/pCMV-7.1 to generate $3 \times$ FNLDD/ pCMV-7.1.

To construct pMXs-I2, the coding sequence of enhanced green fluorescent protein (EGFP) of pMXs-IG [5] was replaced with that of human CD2 antigen (hCD2) [6].

To construct $3 \times$ FNLDD/pMXs-I2, the DNA sequence encoding $3 \times$ FNLDD was cleaved with Sac I and Sal I, blunted, and inserted into the pMXs-I2 vector that was cleaved with EcoR I and Not I and blunted.

All PCR-derived DNA sequences were verified by DNA sequencing.

\subsection{Cell Lines}

293T was maintained in DMEM supplemented with 10\% fetal calf serum (FCS). Ba/F3 [7]-derived cells were maintained in RPMI-1640 supplemented with 10\% FCS, $10 \mathrm{mM}$ Hepes $(\mathrm{pH} 7.2), 1 \times$ non-essential amino acid, 1 $\mathrm{mM}$ sodium pyruvate, $5 \mathrm{nM}$ 2-mercaptoethanol, $1 \mathrm{ng} / \mathrm{ml}$ interleukin-3.

$1 \times 10^{7}$ of $\mathrm{Ba} / \mathrm{F} 3$ were transfected with $\mathrm{Mlu}$ I-digested pGL3C-Neo-cHS4c $\times 24-L e x A \times 2$ [3] $(100 \mu \mathrm{g})$ together with the hygromycin resistance gene $(3 \mu \mathrm{g})$ by electroporation using Gene Pulser II (Bio-Rad) at $250 \mathrm{~V}, 975$ $\mu \mathrm{F}$. The transfected cells were selected in the presence of hygromycin $(1 \mathrm{mg} / \mathrm{ml})$ to establish the cHS4-core-1.2k cell line. Subsequently, $1 \times 10^{7}$ of cHS4-core- $1.2 \mathrm{k}$ were transfected with Sca I-digested $3 \times$ FNLDD/pCMV-7.1 $(100 \mu \mathrm{g})$ or Hind III-digested FCNLD/pEF $(100 \mu \mathrm{g})$ together with the puromycin resistance gene $(3 \mu \mathrm{g})$ by electroporation. The transfected cells were selected in the presence of hygromycin $(1 \mathrm{mg} / \mathrm{ml})$ and puromycin $(0.6$ $\mu \mathrm{g} / \mathrm{ml})$ to establish the $3 \times \mathrm{FNLDD} / \mathrm{cHS} 4$-core- $1.2 \mathrm{k}$ or FCNLD/cHS4-core-1.2k cell line.

\subsection{Immunoblot Analysis}

Nuclear extracts were prepared with NE-PER Nuclear and Cytoplasmic Extraction Reagents (Pierce). Immunoblot analysis was performed as described before [6]. AntiFLAG M2 Ab was purchased from Sigma-Aldrich.

\subsection{Flowcytometry}

$2 \mu \mathrm{g}$ of pMXs-I2 or $3 \times \mathrm{FNLDD} / \mathrm{pMXs}-\mathrm{I} 2$ was transfected into $1 \times 10^{6}$ of $293 \mathrm{~T}$ cells with Lipofectamine 2000 (Invitrogen). Two days after transfection, cells were har- vested and stained with phycoerythrin (PE)-conjugated anti-hCD2 Ab (BD Biosciences). Subsequently, cells were intracellularly stained with fluorescein isothiocyanate (FITC)-conjugated anti-FLAG M2 (Sigma-Aldrich) using the Fixation/Permeabilization and Permeabilization buffer set (eBioscicence). Flowcytometric analysis was performed on FACS Calibur (BD Biosciences), and data was analyzed with FlowJo software (TreeStar).

\subsection{Chromatin Preparation and iChIP}

Cells $\left(4 \times 10^{6}\right)$ were fixed with $1 \%$ formaldehyde at $37^{\circ} \mathrm{C}$ for $5 \mathrm{~min}$. The chromatin fraction was extracted and fragmented ( $2 \mathrm{kbp}$-long on average) by sonication and subjected to iChIP as described previously [3]. The DNA purified by phenol-chloroform extraction and ethanol precipitation was used as a template for real-time PCR with Power SYBR Green PCR Master Mix (Applied Biosystems) using the Applied Biosystems 7900HT Fast Real-Time PCR System. PCR cycles were as follows: heating at $50^{\circ} \mathrm{C}$ for $2 \mathrm{~min}$ followed by $95^{\circ} \mathrm{C}$ for $10 \mathrm{~min}$; 40 cycles of $95^{\circ} \mathrm{C}$ for $15 \mathrm{sec}$ and $60^{\circ} \mathrm{C}$ for $1 \mathrm{~min}$. The primers used in this experiment are LexA-N2 (26572) (5'-ttctctatcgataggtacctcg-3') and LexA-C (26573) (5'-tct attcagcggatctcgagcg-3').

\section{RESULTS AND DISCUSSION}

\subsection{Generation of the Expression System of 3×FNLDD}

For iChIP analysis, we have used the FCNLD protein [2] consisting of $2 \times$ FLAG tags, a tobacco etch virus (TEV) protease cleavage site, calmodulin-binidng peptide, the NLS of SV40-T-antigen, and LexA DB. To generate more efficient tagged LexA proteins for iChIP analysis, we constructed a plasmid expressing the $3 \times$ FNLDD protein consisting of $3 \times$ FLAG tags, the NLS of SV40T-antigen, and DB as well as the dimerization domain of LexA (Figure 1(a)). To increase efficiency of immunoprecipitation, $3 \times$ FLAG tags were used instead of $2 \times$ FLAG tags. In addition, we removed the TEV protease cleavage site and calmodulin-binding peptide because efficiency of cleavage by TEV was low in crosslinked chromatin (data not shown).

The pMXs-I2 vector expressing hCD2 or $3 \times \mathrm{FNLDD} /$ pMXs-I2 bicistronically expressing $3 \times$ FNLDD and hCD2 was transfected into $293 \mathrm{~T}$ cells. Nuclear extracts were prepared and subjected to immunoblot analysis using anti-FLAG Ab. As shown in Figure 1(b), expression of $3 \times$ FNLDD was detected.

Next, we examined expression of $3 \times$ FNLDD in individual cells by flowcytometry. 293T cells transfected with pMXs-I2 or $3 \times$ FNLDD/pMXs-I2 were stained with $\mathrm{PE}-$ conjugated anti-hCD2 $\mathrm{Ab}$ and subsequently intracel- 
lularly stained with FITC-conjugated anti-FLAG Ab. As shown in Figure 1(c), expression of $3 \times$ FNLDD was clearly detected on hCD2 $(+)$ cells.

\subsection{Efficient Isolation of Specific Genomic Regions by iChIP with $3 \times$ FNLDD}

Next, we examined efficiency of iChIP with $3 \times$ FNLDD. To this end, we first established a cell line, cHS4-core$1.2 \mathrm{k}$, by transfection of the pGL3C-Neo-cHS4c $\times 24-$ LexA $\times 2$ plasmid possessing 2 copies of the cHS4c $\times$ 12-LexA cassette, in which $8 \times$ repeats of the LexA DNA-binding sequence was flanked at each side by six copies of the core sequence of chicken HS4 insulator (cHS4-core) (Figure 2(a)). The cHS4-core-1.2k cell line retained about 100 copies of the cHS4c $\times 12$-LexA cassette in the genome (data not shown), thus about 1200 copies of the cHS4-core sequence are integrated in the genome. We subsequently transfected the FCNLD/pEF or the $3 \times \mathrm{FNLDD} / \mathrm{pCMV}-7.1$ plasmid into the cHS4core-1.21 cell line to express FCNLD or $3 \times$ FNLDD, respectively. The chromatin fraction was prepared from the stable cell lines expressing FCNLD or $3 \times$ FNLDD and subjected to iChIP for isolation of cHS4-core as a target region. Isolation efficiency of cHS4-core was evaluated by detection of the LexA-binding elements in real-time PCR (Figure 2(a)). Multiple clones expressing FCNLD or $3 \times$ FNLDD were analyzed to obtain clones showing high iChIP efficiency. Figure 2(b) shows \% input of the LexA-binding elements purified by iChIP using a representative FCNLD- or $3 \times$ FNLDD-expressing clone. iChIP with anti-FLAG Ab using the FCNLD-expressing clone showed $2.9 \%$ of the input DNA, which is consistent with the data we reported previously [2]. In contrast, the $3 \times$ FNLDD-expressing clone showed $11.3 \%$ of the input, which is several-fold higher than the FCNLD-expressing clone. We observed consistent results using clones established independently (data not shown). In contrast, $\%$ input of the promoter region of the glyceraldehyde 3-phosphate dehydrogenase (GAPDH) gene, a negative control genomic region, was less than $0.1 \%$ in $3 \times$ FNLDDexpressing clones (data not shown), showing that backgrounds of iChIP with $3 \times$ FNLDD are low. These data indicate that $3 \times$ FNLDD would immunoprecipitate the target sequence more efficiently than FCNLD and be useful for efficient isolation of specific genomic regions by iChIP. Increase in the number of the FLAG tag sequence may contribute to increase in efficiency of immunoprecipitation. In addition, addition of the dimerization domain of LexA would help form stable dimers to increase binding affinity/avidity to the LexA elements.

\section{CONCLUSIONS}

In this study, we generated a second generation tagged

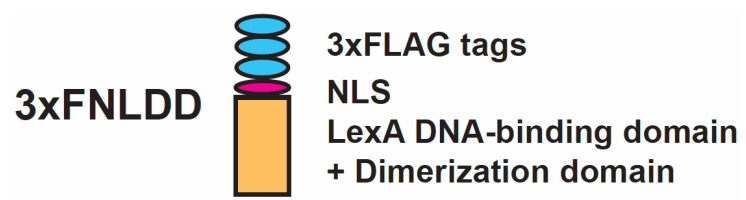

(a)

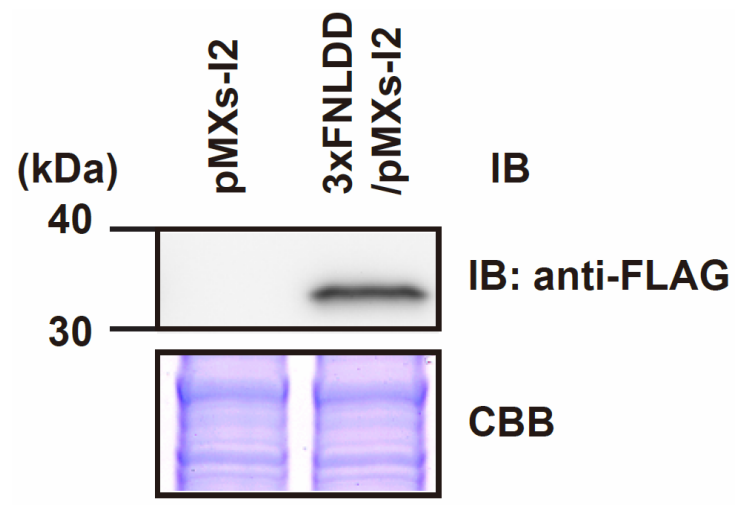

(b)

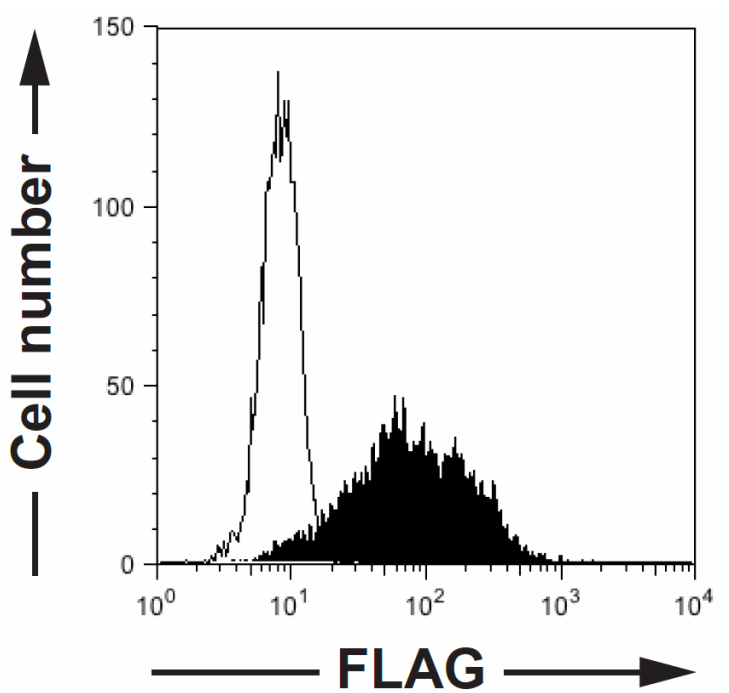

(c)

Figure 1. Scheme of $3 \times$ FNLDD. (a) $3 \times$ FNLDD consists of $3 \times$ FLAG tags, a nuclear localization signal (NLS), the DNA-binding domain (DB) and the dimerization domain of the LexA protein; (b) Expression of $3 \times$ FNLDD in $293 \mathrm{~T}$ cells. Nuclear extracts were subjected to immunoblot analysis with anti-FLAG Ab. The Coomassie Brilliant Blue staining (CBB) is shown as a protein loading control; (c) Flowcytometric detection of $3 \times$ FNLDD. pMXs-I2 or $3 \times \mathrm{FNLDD} / \mathrm{pMXs}-\mathrm{I} 2$ was transfected into 293T cells with Lipofectamine 2000. Two days after transfection, cells were harvested and stained with PEconjugated anti-hCD2 Ab. Subsequently, cells were intracellularly stained with FITC-conjugated anti-FLAG $\mathrm{M} 2$. hCD2 $(+)$ cells were gated to quantify expression levels of $3 \times$ FNLDD. Open: pMXs-I2-trans-fected cells; solid: $3 \times$ FNLDD/pMXs-I2-transfected cells. Mean fluorescent intensity of FLAG: pMXs-I2, 8.48; $3 \times$ FNLDD/ pMXs-IG, 122. Mean fluorescent intensity of hCD2: pMXs-IG, 623; 3×FNLDD/pMXs-IG, 636 . 


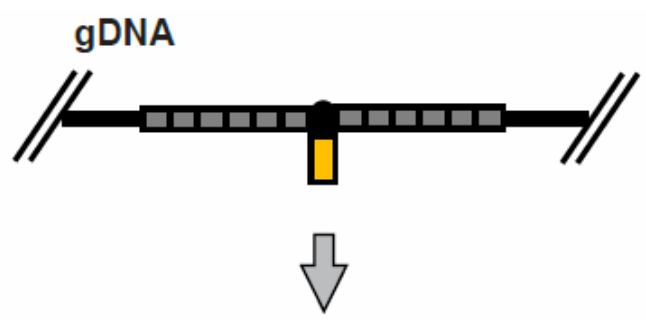

iChIP with anti-FLAG $A b$
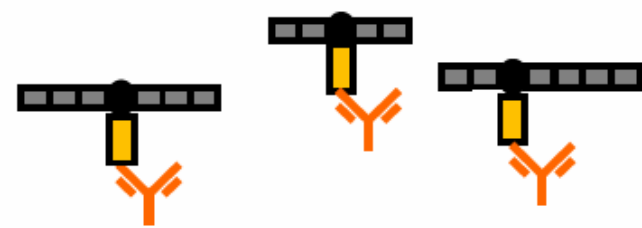

DNA purification

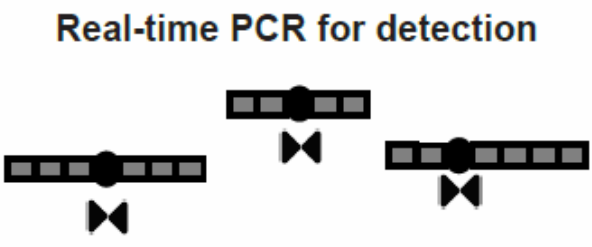

$8 \times$ LexA-binding elements (0.2 kbp) 口 cHS4-core (0.25 kbp)

3xFNLDD or FCNLD

\PCR primers

(a)

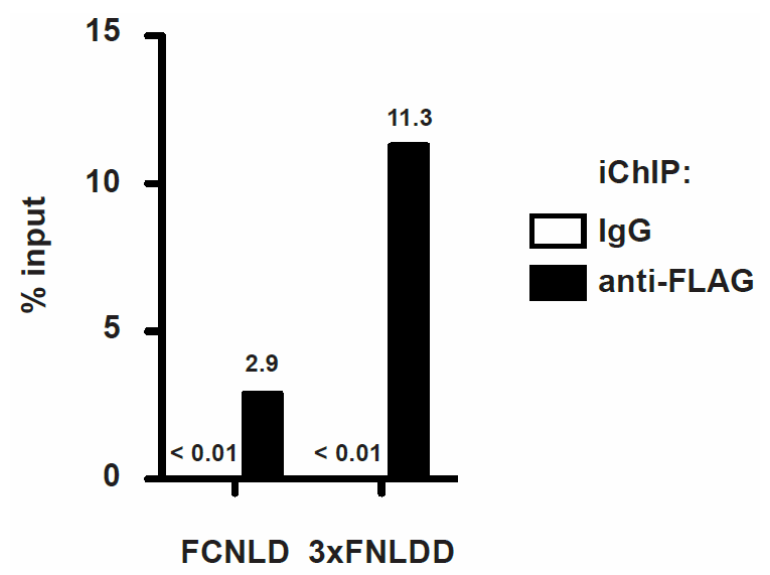

(b)

Figure 2. Efficient isolation of specific genomic regions by iChIP with $3 \times$ FNLDD. (a) Scheme of iChIP in this study. The cHS4c $\times 12$-LexA regions integrated into the genome of $\mathrm{Ba} / \mathrm{F} 3$ cell line were isolated by iChIP with anti-FLAG $\mathrm{Ab}$ and analyzed by real-time PCR with LexA primers; (b) Quantification of the amounts of the immunoprecipitated regions by real-time PCR.
LexA, $3 \times$ FNLDD, for more efficient iChIP analysis. By using $3 \times$ FNLDD, we were able to isolate target genomic regions much more efficiently than by using FCNLD. \% input by using $3 \times$ FNLDD reached more than $10 \%$, which would enable biochemical analysis of specific genomic regions much easier.

In addition, we showed that expression of $3 \times$ FNLDD can be detected and quantified by flowcytometry. Flowcytometric detection of $3 \times$ FNLDD would be useful in easy quantification of its expression levels in individual cells. Taken together, $3 \times$ FNLDD would be a useful tool for iChIP analysis of specific genomic regions.

\section{ACKNOWLEDGEMENTS}

This work was supported by Nakatani Foundation for Advancement of Measuring Technologies in Biomedical Engineering (T.F.), Japan Science and Technology Agency (JST) (H.F.), Grant-in-Aid for Young Scientists (B) (\#22710185) (T.F.), Grant-in-Aid for Scientific Research on Innovative Areas (\#23118516) (T.F.), (\#23114707) (H.F.) from the Ministry of Education, Culture, Sports, Science and Technology of Japan.

\section{REFERENCES}

[1] van Driel, R., Fransz, P.F. and Verschure, P.J. (2003) The eukaryotic genome: A system regulated at different hierarchical levels. Journal of Cell Science, 116, 4067-4075. doi: $10.1242 /$ jes.00779

[2] Hoshino, A. and Fujii, H. (2009) Insertional chromatin immunoprecipitation: A method for isolating specific genomic regions. Journal of Bioscience and Bioengineering, 108, 446-449. doi:10.1016/j.jbiosc.2009.05.005

[3] Fujita, T. and Fujii, H. (2011) Direct idenification of insulator components by insertional chromatin immunoprecipitation. PLoS One, 6, e26109. doi:10.1371/journal.pone.0026109

[4] Bartel, P., Chien, C.T., Sternglanz, R. and Fields, S. (1993) Elimination of false positives that arise in using the two-hybrid system. BioTechniques, 14, 920-924.

[5] Nosaka, T., Kawashima, T., Misawa, K., Ikuta, K., Mui, A.L. and Kitamura, T. (1999) STAT5 as a molecular regulator of proliferation, differentiation and apoptosis in hematopoietic cells. EMBO Journal, 18, 4754-4765. doi:10.1093/emboj/18.17.4754

[6] Hoshino, A., Matsumura, S., Kondo, K., Hirst, J.A. and Fujii, H. (2004) Inducible translocation trap: A system for detecting inducible nuclear translocation. Molecular Cell, 15, 153-159. doi:10.1016/j.molcel.2004.06.017

[7] Palacios, R. and Steinmetz, M. (1985) IL3-dependent mouse clones that express B-220 surface-antigen, contain $\mathrm{Ig}$ genes in germ-line configuration, and generate lymphocytes-B in vivo. Cell, 41, 727-734. doi:10.1016/S0092-8674(85)80053-2 